\title{
The "eSecMed" app: Secure medication through NFC-solutions for a longer, safer \& autonomous life of seniors
}

\author{
Gert Breitfuss \\ evolaris next level $\mathrm{GmbH}$ \\ Hugo-Wolf-Gasse 8-8a, Graz, Austria \\ gert.breitfuss@evolaris.net \\ Stefan Raschhofer \\ evolaris next level $\mathrm{GmbH}$ \\ Hugo-Wolf-Gasse 8-8a, Graz, Austria \\ stefan.raschhofer@evolaris.net \\ Elena Stocker \\ Research Center Pharmaceutical Engineering $\mathrm{GmbH}$ \\ Inffeldgasse 13, Graz, Austria \\ elena.stocker@rcpe.at \\ Carolin Kollegger \\ Infineon Technologies Austria AG \\ Babenbergerstrasse 10, Graz, Austria \\ Carolin.Kollegger-EE@infineon.com
}

\section{Summary}

Within the project "eSecMed-Secure Medication through NFC-Solutions for a longer, safer \& autonomous Life of Seniors" funded by the Austrian Research Promotion Agency (FFG), a consortium of 3 partners developed an intelligent blister with a NFC chip and integrated booster antenna combined with an mHealth smartphone application. The objective of this system was to enhance health adherence/compliance and authentication possibilities of medications, as well as minimising undesirable side effects and harmful interactions. This paper describes the evaluation of the overall system with the main focus on the development of an application prototype. With a qualitative assessment (usability testing method) the present solution was tested in terms of usefulness, ease of use and technology acceptance.

Keywords: usability, mobile application, medical compliance, NFC, mHealth, technology acceptance 


\section{Introduction}

"Medicines will not work if you do not take them" - the WHO stated in 2003, addressing the lack of adherence to prescribed long-term therapies and stressing the fact that only half of all chronically ill patients are compliant to treatment recommendations. [1]

This not only has a negative influence on treatment outcomes but also on the number of cases of avoidable hospitalizations, which are estimated to amount to more than $\$ 100$ billion per year. [2]

Due to the fact that the aging process influences the metabolism and causes certain physical and psychological deficits, elderly persons are more likely to be affected by coexisting chronic diseases. In order to treat these conditions multiple medications are prescribed (polypharmacy) which increase the occurrence of undesired harmful drug interactions. [3]

In 2010 , it was estimated that within the age group of 65 plus $44 \%$ of men and $57 \%$ of women take at least five different medications each week. [4] The probability of adverse drug effects will reach from $13 \%$ when two medications are taken to $58 \%$ for five and rises further as the number of medications increases. [5]

Unexpected side effects can also occur, when counterfeit drugs with incorrect ingredients or dosages thereof are taken. According to estimates of the Food and Drug Administration (FDA) at least $10 \%$ of all medications worldwide are falsified. [6] The proportion of counterfeit drugs even exceeds $50 \%$ in some countries. [7]

In addition, the Counterfeiting Superhighway report, published by the European Alliance for Access to Safe Medicines (EAASM) in 2008, indicated that $63 \%$ of all compounds which were purchased from more than 100 online pharmacies were counterfeit or at least substandard. [8]

Information-and-Communication-Technology (ICT)-based solutions put themselves forward for countering the described situation. Thus, the applicability of Near Field Communication (NFC)-enabled mobile phones to facilitate data management and exchange as well as patient monitoring with the additional benefits of reducing time and errors and the high availability of the technology has been pointed out by a number of authors. $[9,10,11]$

Within the project "eSecMed" funded by the Austrian Research Promotion Agency (FFG), a consortium of 3 partners was assigned with the development of an intelligent blister with an NFC chip and integrated booster antenna combined with an mHealth smartphone application. eSecMed aims to improve patient security through ICT solutions from pharmaceutical manufacturers to the intake of pills by seniors with the ultimate goal to enable seniors to live a longer secure $\&$ autonomous life at home. Therefore the consortium partners defined the following application-oriented goals: 
- To increase the health adherence by application of NFC as a user-friendly technology suitable for seniors, to assist secure medication and to provide additional information for increasing acceptance, usability \& compliance.

- To minimize undesirable side effects and harmful interactions of drugs for seniors by development of a NFC supported virtual medicine cabinet as a smartphone app for mobile devices.

To meet these challenging objectives, the industry partner Infineon (an electronic semi-conductor producer), the Research Center for Pharmaceutical Engineering (RCPE) and the competence center for mobile communication and innovation evolaris joined forces in order to maximize the benefit of the complementary expertise for the target group.

Apart from the objectives mentioned above, also the willingness of seniors to use the new technology plays a key role. Especially during the development of the application concept the Quality by Design $(\mathrm{QbD})$ approach was used. The key aspect of $\mathrm{QbD}$ is the assumption that the desired quality of a product or service can be achieved by taking targeted measures during the design process. To achieve this, it is necessary to gain knowledge about the customer needs as well as the risks that are associated with essential processes. [12] As a preliminary point the user needs were identified by using the Living Lab ${ }^{1}$ approach, where focus groups consisting of chronically ill patients and caregivers were interviewed in the course of several workshops. Also challenges and possible solutions were discussed with experts such as general practitioners and pharmacists. Due to the inclusion of these persons, it was possible to consider the requirements of every actor involved.

This phase was followed by a first testing phase where potential users examined paper prototypes. The feedback of this group in combination with the results from the requirements phase entered the next iteration within the conception phase and provided the foundation for the design of the existing application.

Bearing these facts in mind, the central thesis of the current study is based on the following research question:

- Does the available solution (application and intelligent blister) meet the requirements of seniors in terms of usability and technology acceptance?

\section{The "eSecMed" app}

The main goal of the eSecMed project was the development of an easy to use health application, which acts as a virtual medicine cabinet and the creation of an intelligent pillbox with an NFC chip as well as an integrated booster antenna.

\footnotetext{
${ }^{1}$ Within the evolaris Mobile Living Lab users are being involved in the process of developing mobile communications solutions. Optimization and increase of the acceptance of ICT products and services through user centered design is a main part of the evolaris development process. Since 2008 the evolaris Mobile Living Lab is part of the European Network of Living Labs (ENoLL) and one of three Living Labs in Austria.
} 
By combining these two components, autonomy and safety of elderly patients can be improved. The following features were designed to ensure the aforementioned:

- The 'Medicine Cabinet' is supposed to support patients in taking the right amount of medication at the right time (see third screen on figure 2). For that reason it is possible to incorporate different medicines by simply scanning the blister with the smartphone (NFC enabled). Once this is done, the patient can enter the prescribed dosage, as well as when it has to be taken. This is enhanced by an alert-function, which can be enabled at ones convenience. Above that, every medication in the cabinet offers information on how many pills are left in the blister (including a warning, when the number reaches a certain value), expiration date, last documented intakes, compliance and storage conditions in terms of temperature.

- Documentation of intake: Every time a pill is removed from the blister, a timestamp is created. This timestamp and the number of pills removed at that time are compared to the prescription data in order to determine the patient's compliance. Documentation is conducted automatically every time the user scans the blister. A brief message appears on the screen to inform the user, whether the documentation has been performed successfully or not.

- Check for adverse effects: By utilizing a database, different medicines can be selected to be checked for adverse effects, regardless of whether the former have already been added to the medicine cabinet or not. This is especially crucial when it comes to polypharmacy.

- Check for authenticity of medications: Also by means of a database, the genuineness of medications can be proved (see second screen on figure 2)

- Daily reports are generated which provide information about the patient's compliance. If there are nonconformities with the prescription in terms of dosage or time of intake, they are highlighted in the report to help patients to improve their compliance.

- Medi Buddies: In order to involve caregivers who can support the patient emotionally, the patient is given the possibility to share the daily reports with them.

- It is also possible for the patient to manually enter data, in case the NFCfeature is not performing as designed.

Figure 1 shows the interaction of an NFC-able smartphone with the intelligent blister. When the smart blister and a smartphone, on which the eSecMed application is installed and active, are brought into immediate proximity of each other, the NFC-connection is established and data will be transferred from the blister to the smartphone. The application starts via the start screen (shown in figure 2) from where the user navigates to the required function. 


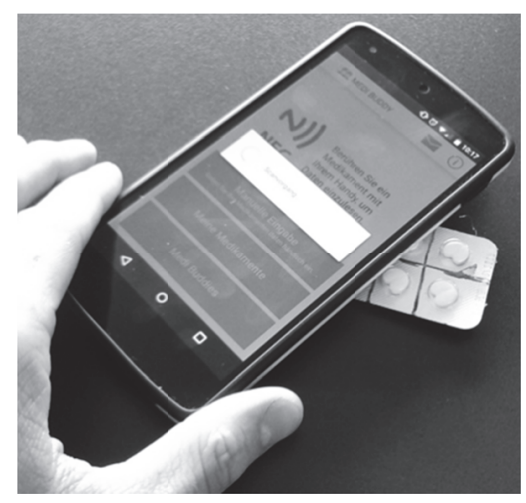

Figure 1: NFC interaction of application and intelligent blister

The application is designed to meet the needs of elderly patients. For that reason during the conception phase we focused on short navigation paths, as little manual input as possible and reasonable font sizes.

The electrical components enable the described smart blister to store a unique identification number and to keep track of temperature as well as resistance values, which allow a detection of how many pills, have been removed so far. By means of NFC technology this data can be retrieved by an accordingly equipped smartphone.
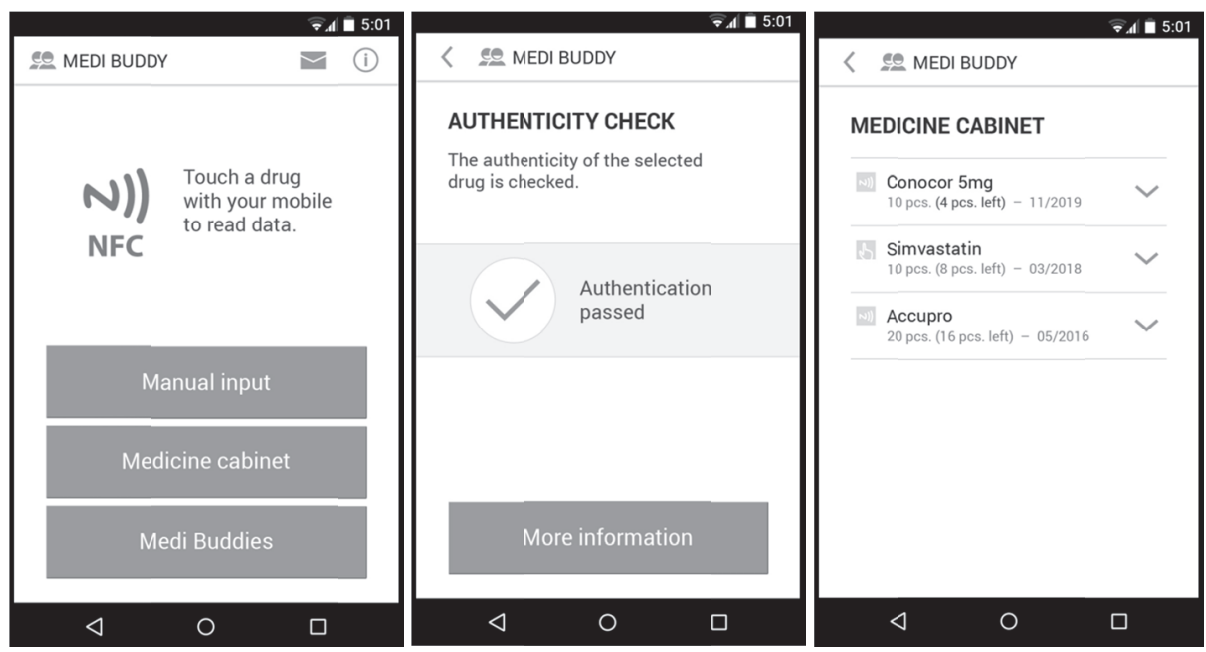

Figure 2: Screen examples 


\section{Method}

This study is based on the method of usability evaluation. Usability is the ease of use and learnability of a human-made object [13]. The primary notion of usability is that an object designed with a generalized users' psychology and physiology in mind is: [14]

- More efficient to use - takes less time to accomplish a particular task

- Easier to learn - operation can be learned by observing the object

- More satisfying to use

As a general rule for delivering a great experience to mobile users, it is important to think like a mobile user.

- What are customers looking for from a mobile experience?

- What goal do they want to achieve? [15]

The "eSecMed" application was tested with 10 Austrian respondents (6 male, 4 female) using a Nexus 5 (Android 4.1.2) who shared the following sociodemographic characteristics:

- Age: 52 - 69 years

- Medical treatment for a longer period of time within the last 12 months

- Owner of a smartphone with experience in using it

- Not using an application within medical context

The project partner evolaris outlined the usability guidelines for the application and the intelligent blister. The basic concept (paper prototype) was evaluated through the evolaris mobile Living Lab. Based on these results and the underlying research question, an appropriate guideline for this study was compiled. The focus lay on the personal opinion of the respondents. Both positive and negative impressions were taken into account. The interviews were analyzed anonymously and serve as a basis for optimizing the application. This guideline has been divided into three areas.

In the first part (pre-test-questionnaire) experiences with medication handling and first impressions were established. In the second part (tasks-use cases) the respondents had to solve specific tasks relating to the functions of the present solution. Finally (post-test-questionnaire) the respondents were asked questions about the usability of the application and the technology acceptance of the entire system.

\section{Results}

The results of the interviews were recorded as follows:

\section{Pre-test: Medication handling in everyday life and first impressions}

All respondents have developed experience and strategies in the handling of their medication in everyday life to a certain degree. Strategies reach from using particular kinds of pillboxes, using the alarm function on their smartphone, daily routine (intake at a certain time at a certain place) or support from their 
partner. Information about adverse effects are obtained hardly ever, the respondents fully trust their doctors and pharmacists. In some cases the patient information leaflet or the internet is consulted.

- 4 out of 10 users were unfamiliar with the term "NFC technology", although the concept of contactless payment via NFC was known by everyone.

- Respondents tried to tap the NFC logo on the home screen to 'activate' the NFC interface.

- 'Medicine cabinet' and 'manual data input' were the first options that struck the eye of the respondents.

- Large size of buttons and fonts were appreciated.

- Elements were found neatly arranged.

\section{Task 1: Put this medication into the virtual cabinet}

Without preceding instruction the act of 'scanning' the pillbox with NFC caused major difficulties among most of the respondents. Some of the subjects handled the smartphone and the blister with extra care as they were afraid of breaking the equipment. Also the time necessary to properly 'scan' the blister was misjudged. This lead the respondents to just tap the blister with the smartphone for a split-second, which was insufficient to complete the 'scanning'-process. Therefore most subjects turned to the option 'manual data input'. Because of the difficulties using NFC the subjective opinion arose that it would take longer to use it than to manually input the data. At the same time entering complicated names of medications using the keyboard of the smartphone was experienced to be cumbersome by some.

Entering the prescription data could be performed without major problems and was used by $80 \%$ of the respondents intuitively. All of the respondents appreciated the amount of information, the clear structure and the information on how many pills are left in the box.

\section{Task 2: Perform an authenticity check}

Most respondents (80\%) expected this feature to be located inside the medicine cabinet. NFC was not used until the feature could be found in the medicine cabinet. The amount of information provided by the authenticity check increased trust in the application among the respondents especially the information on the expiration date. This feature was mostly seen as a nice-to-have feature as the respondents generally trust their pharmacists and would not expect a medication purchased in a pharmacy to be counterfeit.

\section{Task 3: Push out a pill and document the intake}

While one half of the respondents embraced the speed and convenience of the documentation, the other half was insecure whether the documentation was 
completed or conducted successfully. The message to inform them was stated to be displayed not long enough to read completely or it was not noticed at all. Manual documentation was experienced to be tedious. One respondent expressed the wish for a text box for personal notes.

\section{Task 4: Perform a check on adverse effects with two different medications}

Same as with the authenticity check, many respondents looked for this feature inside the medicine cabinet. Also NFC was not used right away. Once used the feature itself was reported to be helpful. Although using color codes was found useful. For some respondents the codes were not entirely clear, as was the difference between a warning and the urge for caution.

\section{Post-Test: General statement about usability and technology acceptance}

The idea of scanning a blister via NFC got a positive rating. All respondents rated the idea of scanning a blister via a smartphone application on a 5 point rating scale ( $1=$ very good, $5=$ not good at all) with 1 (4 respondents) and 2 (4 respondents). In general the usability was rated positive with valuable feedback for improvements in the area of understandability and performance of the scanning function.

\section{Conclusion}

Although the target group knew about the existence of NFC technology, the majority was not familiar with alternative domains of NFC besides payment solutions. Also handling this technology is still an unknown territory and causes fears. However confidence increased rapidly after introducing users to the technology and the correct way of handling it. In this respect, the user recognized the usefulness of the solution.

In addition to the already implemented functions of the NFC chip there are plans for enhancements. Through further miniaturization an integrated battery will be available to realize data logging e.g. temperature and humidity sensing across the entire supply chain to monitor transport conditions. Also integration in fully automated primary and/or secondary packaging process is possible. Furthermore electrochemical sensors could be realized for the instant analysis of saliva or blood. It remains to be seen if pharmaceutical companies and semiconductor manufactures are willing to further develop the prototypical solution in future. 


\section{References}

[1] World Health Organization (WHO). Adherence to long-term therapies: Evidence for Action. Geneva : WHO, 2003

[2] Osterberg, Lars; Blaschke, Terrence. Adherence to medication. // New England Journal of Medicine. 353 (2005), 3; 487-497

[3] Sergi, Guiseppe; De Rui, Marina; Sarti, Silvia; Manzato, Enzo. Polypharmacy in the Elderly - Can comprehensive Geriatric Assessment Reduce Inappropriate Medication Use? // Drugs and Aging. 28 (2011), 7; 509-518.

[4] Woodruff, Kathleen. Preventing Polypharmacy in Older Adults. // American Nurse Today. 5 (2010), 10

[5] Prybys, Katherine; Melville, Kraig; Hanna, Jeahan; Gee, Andrew; Chyka, Peter. Polypharmacy in the elderly: Clinical challenges in emergency practice: Part 1 overview, etiology and drug interactions. // Emergency Medicine Reports, 23(2002); 145-153

[6] Cockburn, Robert; Newton, N. Paul; Agyarko, E. Kyeremateng; Akunyili, Dora; White, J. Nicholas. The global threat of counterfeit drugs: why industry and governments must communicate the dangers. // PLoS Medicine. 2 (2005)

[7] Sfetcu, Nicholas. Health \& Drugs: Disease, Prescription \& Medication. Raleigh : Lulu Press, 2014

[8] European Alliance for Access to Safe Medicines (EAASM). The Counterfeiting Superhighway. Surrey. 1.7.2015. https://lakemedelsverket.se/upload/press/olaga/455_EAASM_counterfeiting_020608.pdf

[9] Benelli, Giuliano; Pozzebon, Alessandro. Near Field Communication and eHealth: Turning a mobile phone into a multipurpose assistant in health scenarios. // CCIS. 52 (2010); 356-368

[10] Marcus, Adam; Davidzon, Guido; Law, Denise; Verma, Namrata; Fletcher, Rich; Khan, Aamir; Sarmenta, Luis. Using NFC-enabled Mobile Phones for Public Health in Developing Countries. // First International Workshop on Near Field Communication, Hagenberg : IEEE, 2009, 30-35

[11] Pinsker Matthias; Schindler, Karin; Hayn, Dieter; Morak, Jürgen; Kastner, Peter; Riedl, Michaela; Ludvik, Bernhard; Schreier, Günter. Experiences Using Mobile Phones as PatientTerminal for Telemedical Home Care and Therapy Monitoring of Patients Suffering from Chronic Diseases. // Lecture Notes in Computer Science. 5105 (2008); 1305-1312

[12] Juran, M. Joseph. Juran on Quality by Design: The New Steps for Planning Quality into Goods and Services. New York : Free Press, 1992

[13] Dirnbauer, Kurt. Usability - Grundlagen, Beispiele, Trends. Vienna : eye.type design, 2000.

[14] Jordan, W. Patrick; Thomas, Bruce; Weerdmeester, A. Bernard. Usability evaluation in industry. London : Taylor \& Francis, 1996

[15] Kim, Y. et al. Protecting Mobile Devices from Adversarial User by Fine-Grained Analysis of User Behavior, Department of Computer Science, Korea Advanced Institute of Science and Technology (KAIST), 2014, Daejeon 305-701, Republic of Korea. http://link.springer.com/ chapter/10.1007/978-3-642-41674-3_63\#page-1 